Research Article; Received: March 16, 2020; Accepted: August 18, 2021

\title{
SPLIT COMPLEX BI-PERIODIC FIBONACCI AND LUCAS NUMBERS
}

\author{
Nazmiye YILMAZ \\ Karamanoğlu Mehmetbey University, Kamil Özdă̆ Science Faculty, \\ Department of Mathematics, Karaman, TURKEY
}

\begin{abstract}
The initial idea of this paper is to investigate the split complex bi-periodic Fibonacci and Lucas numbers by using SCFLN now on. We try to show some properties of SCFLN by taking into account the properties of the split complex numbers. Then, we present interesting relationships between SCFLN.
\end{abstract}

\section{INTRODUCTION}

The literature contains many articles that related to the special number sequences such as Fibonacci, Lucas, Pell ( $[2,3,6,8,14,15,17,18]$ ). One of these articles goes through to the bi-periodic Fibonacci (or, equivalently, generalized Fibonacci) and the bi-periodic Lucas (or, equivalently, generalized Lucas). In [3, 6], the authors introduced and studied bi-periodic Fibonacci $\left\{q_{n}\right\}_{n=0}^{\infty}$ and bi-periodic Lucas $\left\{l_{n}\right\}_{n=0}^{\infty}$ sequences that depend on two real parameters used in a non-linear (piecewise linear) recurrence relation as defined below.

$$
\begin{gathered}
q_{n}=a^{1-\xi(n)} b^{\xi(n)} q_{n-1}+q_{n-2}, \quad n \geq 2, \\
l_{n}=a^{\xi(n)} b^{1-\xi(n)} l_{n-1}+l_{n-2}, \quad n \geq 2,
\end{gathered}
$$

where $a$ and $b$ are any two nonzero real numbers, $q_{0}=0, q_{1}=1, l_{0}=2, l_{1}=a$ and the condition $\xi(n)=n-2\left\lfloor\frac{n}{2}\right\rfloor$ can be read as

$$
\xi(n)=\left\{\begin{array}{ll}
0, & n \text { is even } \\
1, & n \text { is odd }
\end{array} .\right.
$$

Furthermore, the authors in the references [3], 6] and [18] gave so many properties on the bi-periodic Fibonacci and bi-periodic Lucas sequences as in the following:

2020 Mathematics Subject Classification. 11B39, 17A45.

Keywords. Bi-periodic Fibonacci number, bi-periodic Lucas number, generalized Fibonacci number, split complex number.

๑ yilmaznzmy@gmail.com

(D) 0000-0002-7302-2281.

(C)2022 Ankara University Communications Faculty of Sciences University of Ankara Series A1 Mathematics and Statistics 
- The Binet formulas are given by

$$
q_{n}=\frac{a^{1-\xi(n)}}{(a b)^{\left\lfloor\frac{n}{2}\right\rfloor}}\left(\frac{\alpha^{n}-\beta^{n}}{\alpha-\beta}\right)
$$

and

$$
l_{n}=\frac{a^{\xi(n)}}{(a b)^{\left\lfloor\frac{n+1}{2}\right\rfloor}}\left(\alpha^{n}+\beta^{n}\right),
$$

where the condition $\xi(n)$ is depend to definition in equation (3) and $\alpha, \beta$ are roots of the characteristic equation of $\lambda^{2}-a b \lambda-a b=0$.

- The generating functions for the bi-periodic Fibonacci and the bi-periodic Lucas sequences with odd and even subscripted are

$$
\sum_{m=0}^{\infty} q_{2 m+1} x^{2 m+1}=\frac{x-x^{3}}{1-(a b+2) x^{2}+x^{4}}, \sum_{m=0}^{\infty} q_{2 m} x^{2 m}=\frac{a x^{2}}{1-(a b+2) x^{2}+x^{4}},
$$

and

$$
\sum_{m=0}^{\infty} l_{2 m+1} x^{2 m+1}=\frac{a x+a x^{3}}{1-(a b+2) x^{2}+x^{4}}, \sum_{m=0}^{\infty} l_{2 m} x^{2 m}=\frac{2-(a b+2) x^{2}}{1-(a b+2) x^{2}+x^{4}} .
$$

- The bi-periodic Fibonacci and the bi-peridoic Lucas sequences provide the equations

$$
\begin{gathered}
l_{n}=q_{n-1}+q_{n+1} \\
(a b+4) q_{n}=l_{n-1}+l_{n+1},
\end{gathered}
$$

and

$$
\begin{gathered}
q_{m+n}=\frac{1}{2}\left[\left(\frac{b}{a}\right)^{\xi(m+1) \xi(n)} q_{m} l_{n}+\left(\frac{b}{a}\right)^{\xi(m) \xi(n+1)} q_{n} l_{m}\right] \\
l_{m+n}=\frac{1}{2}\left[\left(a^{2} b^{2}+4 a b\right)\left(\frac{1}{a^{2}}\right)^{\xi(m+1) \xi(n+1)}\left(\frac{1}{a b}\right)^{1-\xi(m+1) \xi(n+1)} q_{m} q_{n}+\left(\frac{b}{a}\right)^{\xi(m) \xi(n)} l_{m} l_{n}\right],
\end{gathered}
$$

and

$$
\begin{gathered}
q_{-n}=(-1)^{n-1} q_{n}, \\
l_{-n}=(-1)^{n} l_{n} .
\end{gathered}
$$

On the other hand, split complex numbers have applications in different areas of mathematics and theoretical physics. A split complex number (or hyperbolic number, also perplex number, double number) has two real number components $a$ and $b$, and the set of split complex numbers is

$$
\mathbb{H}=\left\{x=a+h b: \quad h^{2}=1, a, b \in \mathbb{R}\right\} .
$$


The split complex ring $\mathbb{H}$ is a bidimensional Clifford algebra, look at 10 for details. Also, split complex numbers are useful for measuring distances in the Lorentz spacetime plane (you can examine $[12]$ ). The addition and multiplication of any two split complex numbers such as $x=a+h b, y=c+h d$ are defined by

$$
x+y=a+c+h(b+d) \text { and } x y=a c+b d+h(a d+b c) .
$$

It is clear that this algebra of split complex number is commutative. The conjugate and norm of $x$ are enounced by

$$
\bar{x}=a-b h, \quad x \bar{x}=a^{2}-b^{2} .
$$

For more information on split complex numbers, see for example, 4], 7], [9], 10], 11] and 12$]$.

Many researchs activities can be seen in resent years studies on split complex(or hyperbolic) Fibonacci, Lucas, Jacobsthal and Tribonacci numbers (see [1], [5], 13], [16]). For example, in [1], it was investigated some properties of the split complex Fibonacci numbers are defined as $\tilde{F}_{n}=F_{n}+h F_{n+1}$.

\section{The SPlit COMPLEX BI-PERIODIC FibONACCI AND LuCAS NUMBERS}

The objective of this paper is to define split complex bi-periodic Fibonacci and Lucas numbers(SCFLN) with a different aspect. In this part, we introduce the SCFLN that generalize split complex Fibonacci, split complex Lucas, split complex Pell and split complex Pell-Lucas numbers. We give some properties of the SCFLN such as the Binet formulas, the generating functions, sums, binomial sums of the SCFLN. We also present the Catalan, Cassini, D'Ocagne and other identities of the SCFLN.

Definition 1. The split complex bi-periodic Fibonacci $\left(\tilde{q}_{n}\right)$ and Lucas $\left(\tilde{l}_{n}\right)$ numbers are defined by

$$
\tilde{q}_{n}=q_{n}+h a^{1-\xi(n)} b^{\xi(n)} q_{n+1}, \quad \tilde{q}_{0}=h a, \quad \tilde{q}_{1}=h a b+1
$$

and

$$
\tilde{l}_{n}=l_{n}+h a^{\xi(n)} b^{1-\xi(n)} l_{n+1}, \quad \tilde{l}_{0}=h a b+2, \tilde{l}_{1}=h\left(a^{2} b+2 a\right)+a
$$

where $n \in \mathbb{N}, h^{2}=1, \xi(n)$ is as defined the equation (3) and $q_{n}, l_{n}$ are the bi-periodic Fibonacci and Lucas numbers, respectively.

It can be easily shown that

$$
\tilde{q}_{n}= \begin{cases}a \tilde{q}_{n-1}+\tilde{q}_{n-2}, & \text { if } n \text { is even } \\ b \tilde{q}_{n-1}+\tilde{q}_{n-2}, & \text { if } n \text { is odd }\end{cases}
$$

and

$$
\tilde{l}_{n}=\left\{\begin{array}{ll}
b \tilde{l}_{n-1}+\tilde{l}_{n-2}, & \text { if } n \text { is even } \\
a \tilde{l}_{n-1}+\tilde{l}_{n-2}, & \text { if } n \text { is odd }
\end{array} .\right.
$$


From the equations $(12),(13), 17)$ and $(18)$, the SCFLN with negative subscripts are defined by

$$
\tilde{q}_{-n}=\left\{\begin{array}{ll}
-a \tilde{q}_{-(n-1)}+\tilde{q}_{-(n-2)}, & \text { if } n \text { is even } \\
-b \tilde{q}_{-(n-1)}+\tilde{q}_{-(n-2)}, & \text { if } n \text { is odd }
\end{array},\right.
$$

and

$$
\tilde{l}_{-n}=\left\{\begin{array}{ll}
-b \tilde{l}_{-(n-1)}+\tilde{l}_{-(n-2)}, & \text { if } n \text { is even } \\
-a \tilde{l}_{-(n-1)}+\tilde{l}_{-(n-2)}, & \text { if } n \text { is odd }
\end{array},\right.
$$

where $n \in \mathbb{N}$.

After all, we give the following Table 1. This table show that the first few SCFLN with positive and negative subscripts.

TABLE 1. The first few SCFLN with positive and negative subscripts.

\begin{tabular}{|c|c|c|}
\hline$n$ & $\tilde{q}_{n}$ & $\tilde{l}_{n}$ \\
\hline-4 & $h\left(a^{2} b+a\right)-a^{2} b-2 a$ & $h\left(a^{2} b+3 a\right)-a^{2} b^{2}+4 a b+2$ \\
\hline-3 & $-h a b+a b+1$ & $h\left(a^{2} b+2 a\right)-a^{2} b-3 a$ \\
\hline-2 & $h a-a$ & $-h a b+a b+2$ \\
\hline-1 & 1 & $2 h a-a$ \\
\hline 0 & $h a$ & $h a b+2$ \\
\hline 1 & $h a b+1$ & $h\left(a^{2} b+2 a\right)+a$ \\
\hline 2 & $h\left(a^{2} b+a\right)+a$ & $h\left(a^{2} b^{2}+3 a b\right)+a b+2$ \\
\hline 3 & $h\left(a^{2} b^{2}+2 a b\right)+a b+1$ & $h\left(a^{3} b^{2}+4 a^{2} b+2 a\right)+a^{2} b+3 a$ \\
\hline 4 & $h\left(a^{3} b^{2}+3 a^{2} b+a\right)+a^{2} b+2 a$ & $h\left(a^{3} b^{3}+5 a^{2} b^{2}+5 a b\right)+a^{2} b^{2}+4 a b+2$ \\
\hline
\end{tabular}

Now, we give the Binet formulas for the SCFLN and so find some well-known mathematical properties.

Theorem 1. For any integer n, the Binet formulas for the SCFLN are

$$
\tilde{q}_{n}=\frac{a^{1-\xi(n)}}{(a b)^{\left\lfloor\frac{n}{2}\right\rfloor}}\left(\frac{\tilde{\alpha} \alpha^{n}-\tilde{\beta} \beta^{n}}{\alpha-\beta}\right)
$$

and

$$
\tilde{l}_{n}=\frac{a^{\xi(n)}}{(a b)^{\left\lfloor\frac{n+1}{2}\right\rfloor}}\left(\tilde{\alpha} \alpha^{n}+\tilde{\beta} \beta^{n}\right)
$$

where $\alpha, \beta$ are as the equation (4) and $\tilde{\alpha}=1+h \alpha, \tilde{\beta}=1+h \beta$.

Proof. It can easily established by using the Definition 1 and the Equations (4), (5).

The generating functions of the SCFLN are given in the following theorem. 
Theorem 2. For the SCFLN, we have the generating functions

i) $\sum_{n=0}^{\infty} \tilde{q}_{n} x^{n}=\frac{h\left(a b x+a-a x^{2}\right)+x+a x^{2}-x^{3}}{1-(a b+2) x^{2}+x^{4}}$,

ii) $\sum_{n=0}^{\infty} \tilde{l}_{n} x^{n}=\frac{h\left(a b+a^{2} b x+2 a x+a b x^{2}-2 a x^{3}\right)+2+a x-a b x^{2}-2 x^{2}+a x^{3}}{1-(a b+2) x^{2}+x^{4}}$.

Proof.

i) Let $f(x)=\sum_{n=0}^{\infty} \tilde{q}_{n} x^{n}$. From the Definition 11, we have

$$
\begin{aligned}
f(x) & =\sum_{n=0}^{\infty}\left(h a^{1-\xi(n)} b^{\xi(n)} q_{n+1}+q_{n}\right) x^{n} \\
& =h b \sum_{n=0}^{\infty} q_{2 n+2} x^{2 n+1}+h a \sum_{n=0}^{\infty} q_{2 n+1} x^{2 n}+\sum_{n=0}^{\infty} q_{n} x^{n} .
\end{aligned}
$$

By considering the Equation (6), we obtain

$$
f(x)=\frac{h a b x}{1-(a b+2) x^{2}+x^{4}}+\frac{h a\left(1-x^{2}\right)}{1-(a b+2) x^{2}+x^{4}}+\frac{x+a x^{2}-x^{3}}{1-(a b+2) x^{2}+x^{4}},
$$

as needed.

ii) Similarly, we obtain equation in $i$.

Next, we give the formulas which give the summations and binomial sums of the SCFLN.

Theorem 3. For $n \geq 0$, the following formulas are true:
i) $\sum_{i=0}^{n} a^{\xi(i)}(a b)^{\left\lfloor\frac{i}{2}\right\rfloor} \tilde{q}_{i}=\frac{a^{\xi(n)}(a b)^{\left\lfloor\frac{n}{2}\right\rfloor+1} \tilde{q}_{n}+a^{\xi(n+1)}(a b)^{\left\lfloor\frac{n+1}{2}\right\rfloor} \tilde{q}_{n+1}-a-h a}{2 a b-1}$,
ii) $\sum_{i=0}^{n} a^{-\xi(i)}(a b)^{\left\lfloor\frac{i+1}{2}\right\rfloor} \tilde{l}_{i}=\frac{\left.a^{-\xi(n)}(a b)^{\left\lfloor\frac{n+1}{2}\right\rfloor+1} \tilde{l}_{n}+a^{-\xi(n+1)}(a b)^{\left\lfloor\frac{n+2}{2}\right.}\right\rfloor \tilde{l}_{n+1}-2+a b-3 h a b}{2 a b-1}$,
iii) $\sum_{i=0}^{n}\left(\begin{array}{c}n \\ i\end{array}\right) a^{\xi(i)}(a b)^{\left\lfloor\frac{i}{2}\right\rfloor} \tilde{q}_{i}=\tilde{q}_{2 n}$, $\sum_{i=0}^{n}\left(\begin{array}{c}n \\ i\end{array}\right) a^{\xi(i+1)}(a b)^{\left\lfloor\frac{i+1}{2}\right\rfloor} \tilde{q}_{i+1}=a \tilde{q}_{2 n+1}$,
iv) $\sum_{i=0}^{n}\left(\begin{array}{c}n \\ i\end{array}\right) a^{\xi(i+1)}(a b)^{\left\lfloor\frac{i+1}{2}\right\rfloor} \tilde{l}_{i}=a \tilde{l}_{2 n}$, $\sum_{i=0}^{n}\left(\begin{array}{c}n \\ i\end{array}\right) a^{\xi(i)}(a b)^{\left\lfloor\frac{i+2}{2}\right\rfloor-1} \tilde{l}_{i+1}=\tilde{l}_{2 n+1}$,

Proof. We will prove the parts $i$ and $i v$, since the proof of the others can be done similarly with them. 
i) The proof will be handled just the outcome of Theorem 11. Thus, we consider:

$$
\begin{aligned}
\sum_{i=0}^{n} a^{\xi(i)}(a b)^{\left\lfloor\frac{i}{2}\right\rfloor} \tilde{q}_{i} & =\sum_{i=0}^{n} a \frac{\tilde{\alpha} \alpha^{i}-\tilde{\beta} \beta^{i}}{\alpha-\beta} \\
& =\frac{a \tilde{\alpha}}{\alpha-\beta}\left(\frac{\alpha^{n+1}-1}{\alpha-1}\right)+\frac{a \tilde{\beta}}{\alpha-\beta}\left(\frac{\beta^{n+1}-1}{\beta-1}\right) \\
& =\frac{a}{\alpha-\beta}\left[\frac{\tilde{\alpha}\left(\alpha^{n+1} \beta-\alpha^{n+1}-\beta+1\right)-\tilde{\beta}\left(\beta^{n+1} \alpha-\beta^{n+1}-\alpha+1\right)}{\alpha \beta-\alpha-\beta+1}\right] .
\end{aligned}
$$

At this point, by rearragement the last equality by using the equalities $\alpha \beta=-a b$ and $\alpha+\beta=a b$, we give

$$
\begin{aligned}
& \sum_{i=0}^{n} a^{\xi(i)}(a b)^{\left\lfloor\frac{i}{2}\right\rfloor} \tilde{q}_{i}=\frac{-a^{2} b}{1-2 a b} \tilde{q}_{n} \frac{(a b)^{\left\lfloor\frac{n}{2}\right\rfloor}}{a^{1-\xi(n)}}-\frac{a}{1-2 a b} \tilde{q}_{n+1} \frac{(a b)^{\left\lfloor\frac{n+1}{2}\right\rfloor}}{a^{1-\xi(n+1)}} \\
& -\frac{a}{1-2 a b} \frac{\beta(1+h \alpha)-\alpha(1+h \beta)}{\alpha-\beta}+\frac{a}{1-2 a b} h \\
& =\frac{a^{\xi(n)}(a b)^{\left\lfloor\frac{n}{2}\right\rfloor+1} \tilde{q}_{n}+a^{\xi(n+1)}(a b)^{\left\lfloor\frac{n+1}{2}\right\rfloor} \tilde{q}_{n+1}-a-h a}{2 a b-1} .
\end{aligned}
$$

iv) From Theorem 1, we have

$$
\begin{aligned}
\sum_{i=0}^{n}\left(\begin{array}{c}
n \\
i
\end{array}\right) a^{\xi(i+1)}(a b)^{\left\lfloor\frac{i+1}{2}\right\rfloor} \tilde{l}_{i} & =\sum_{i=0}^{n}\left(\begin{array}{c}
n \\
i
\end{array}\right) a^{1-\xi(i)}(a b)^{\left\lfloor\frac{i+1}{2}\right\rfloor} \frac{a^{\xi(i)}}{(a b)^{\left\lfloor\frac{i+1}{2}\right\rfloor}}\left(\tilde{\alpha} \alpha^{i}+\tilde{\beta} \beta^{i}\right) \\
& =a \tilde{\alpha}(1+\alpha)^{n}+a \tilde{\beta}(1+\beta)^{n} .
\end{aligned}
$$

By using the equalities $a b(1+\alpha)=\alpha^{2}$ and $a b(1+\beta)=\beta^{2}$, we get

$$
\begin{aligned}
\sum_{i=0}^{n}\left(\begin{array}{c}
n \\
i
\end{array}\right) a^{\xi(i+1)}(a b)^{\left\lfloor\frac{i+1}{2}\right\rfloor} \tilde{l}_{i} & =\frac{a}{(a b)^{n}}\left(\tilde{\alpha} \alpha^{2 n}+\tilde{\beta} \beta^{2 n}\right) \\
& =a \tilde{l}_{2 n} .
\end{aligned}
$$

Similarly, we obtain

$$
\begin{aligned}
\sum_{i=0}^{n}\left(\begin{array}{c}
n \\
i
\end{array}\right) a^{\xi(i)}(a b)^{\left\lfloor\frac{i+2}{2}\right\rfloor-1} \tilde{l}_{i+1} & =\sum_{i=0}^{n}\left(\begin{array}{c}
n \\
i
\end{array}\right) \frac{a}{a b}\left(\tilde{\alpha} \alpha^{i+1}+\tilde{\beta} \beta^{i+1}\right) \\
& =\frac{a \alpha}{a b} \tilde{\alpha}(1+\alpha)^{n}+\frac{a \beta}{a b} \tilde{\beta}(1+\beta)^{n} \\
& =\frac{a}{(a b)^{n+1}}\left(\tilde{\alpha} \alpha^{2 n+1}+\tilde{\beta} \beta^{2 n+1}\right) \\
& =\tilde{l}_{2 n+1} .
\end{aligned}
$$


Theorem 4. The relations of between the SCFLN are

i)

$$
\begin{aligned}
\tilde{q}_{n+r} & =\left(\frac{b}{a}\right)^{\xi(n+1) \xi(r)} q_{r+1}\left(h a^{1-\xi(n)} b^{\xi(n)} q_{n+1}+q_{n}\right) \\
& +\left(\frac{b}{a}\right)^{\xi(r+1) \xi(n)} q_{r}\left(h a^{\xi(n)} b^{1-\xi(n)} q_{n}+q_{n-1}\right)
\end{aligned}
$$

ii)

$$
\begin{aligned}
\tilde{l}_{n+r} & =\left(\frac{b}{a}\right)^{\xi(n) \xi(r)} q_{r+1}\left(h a^{\xi(n)} b^{1-\xi(n)} l_{n+1}+l_{n}\right) \\
& +\left(\frac{b}{a}\right)^{\xi(n+1) \xi(r+1)} q_{r}\left(h a^{1-\xi(n)} b^{\xi(n)} l_{n}+l_{n-1}\right),
\end{aligned}
$$

iii) $\tilde{q}_{-n}=(-1)^{n} \tilde{q}_{n-2}+(-1)^{n+1} p_{n-1}$,

iv) $\tilde{l}_{-n}=(-1)^{n-1} \tilde{l}_{n-2}+(-1)^{n}(a b+4) q_{n-1}$,

v) $\tilde{q}_{n}=\tilde{l}_{n+1}+\tilde{l}_{n-1}$,

vi) $(a b+4) \tilde{l}_{n}=\tilde{q}_{n+1}+\tilde{q}_{n-1}$,

where $n, r \in \mathbb{N}$.

Proof. i) Using the Equation (1), Definition 1. Table 1 and iterative method, it was obtained

$$
\begin{aligned}
\tilde{q}_{n} & =h a^{1-\xi(n)} b^{\xi(n)} q_{n+1}+q_{n}, \\
\tilde{q}_{n+1} & =(h a b+1) q_{n+1}+h a^{\xi(n)} b^{1-\xi(n)} q_{n}, \\
\tilde{q}_{n+2} & =a^{1-\xi(n)} b^{\xi(n)}(h a b+1+h) q_{n+1}+(h a b+1) q_{n}, \\
\tilde{q}_{n+3} & =\left(h a^{2} b^{2}+a b+2 h a b+1\right) q_{n+1}+h a^{\xi(n)} b^{1-\xi(n)}(h a b+1+h) q_{n}, \\
& \vdots \\
\tilde{q}_{n+r} & =\left(\frac{b}{a}\right)^{\xi(n+1) \xi(r)} q_{r+1}\left(h a^{1-\xi(n)} b^{\xi(n)} q_{n+1}+q_{n}\right) \\
& +\left(\frac{b}{a}\right)^{\xi(r+1) \xi(n)} q_{r}\left(h a^{\xi(n)} b^{1-\xi(n)} q_{n}+q_{n-1}\right) .
\end{aligned}
$$

ii) Using the Equation (2), Definition 1, Table 1 and iterative method, it was obtained

$$
\begin{aligned}
\tilde{l}_{n} & =h a^{\xi(n)} b^{1-\xi(n)} l_{n+1}+l_{n}, \\
\tilde{l}_{n+1} & =(h a b+1) l_{n+1}+h a^{1-\xi(n)} b^{\xi(n)} l_{n}, \\
\tilde{l}_{n+2} & =a^{\xi(n)} b^{1-\xi(n)}(h a b+1+h) l_{n+1}+(h a b+1) l_{n},
\end{aligned}
$$




$$
\begin{aligned}
\tilde{l}_{n+3} & =\left(h a^{2} b^{2}+a b+2 h a b+1\right) l_{n+1}+h a^{1-\xi(n)} b^{\xi(n)}(h a b+1+h) l_{n} \\
& \vdots \\
\tilde{l}_{n+r} & =\left(\frac{b}{a}\right)^{\xi(n) \xi(r)} q_{r+1}\left(h a^{\xi(n)} b^{1-\xi(n)} l_{n+1}+l_{n}\right) \\
& +\left(\frac{b}{a}\right)^{\xi(n+1) \xi(r+1)} q_{r}\left(h a^{1-\xi(n)} b^{\xi(n)} l_{n}+l_{n-1}\right) .
\end{aligned}
$$

iii) By taking account of the Definition 11. Equations (4), (12) and $(19)$, it was obtained

$$
\begin{aligned}
\tilde{q}_{-n} & =(-1)^{n-1} \frac{a^{1-\xi(n)}}{(a b)^{\left\lfloor\frac{n}{2}\right\rfloor}}\left(\frac{\alpha^{n}-\beta^{n}}{\alpha-\beta}\right)+(-1)^{n} h \frac{a b^{\xi(n)}}{(a b)^{\left\lfloor\frac{n-1}{2}\right\rfloor}}\left(\frac{\alpha^{n-1}-\beta^{n-1}}{\alpha-\beta}\right) \\
& =(-1)^{n-1} q_{n}+(-1)^{n} h \frac{a^{1-\xi(n)}}{(a b)^{\left\lfloor\frac{n}{2}\right\rfloor-1}}\left(\frac{\alpha^{n-1}-\beta^{n-1}}{\alpha-\beta}\right)+(-1)^{n} q_{n-2}-(-1)^{n} q_{n-2} \\
& =(-1)^{n} \tilde{q}_{n-2}+(-1)^{n+1} l_{n-1} .
\end{aligned}
$$

$i v$ ) The proof can be done quite similarly as the part iii by using the Definition 1. Equations (5), (13) and (20).

$v$ ) The result is obtained by using Definition 1 and Equation (8). That is, we have

$$
\begin{aligned}
\tilde{l}_{n+1}+\tilde{l}_{n-1} & =h a^{\xi(n+1)} b^{1-\xi(n+1)} l_{n+2}+l_{n+1}+h a^{\xi(n-1)} b^{1-\xi(n-1)} l_{n}+l_{n-1} \\
& =h a^{\xi(n-1)} b^{1-\xi(n-1)} q_{n+1}+q_{n} \\
& =\tilde{q}_{n}
\end{aligned}
$$

as required.

$v i)$ The proof can be done quite similarly as the part $v$ by using Definition 1 and Equation (9).

Following Theorem gives Catalan's identities for the SCFLN;

Theorem 5. For $n, r \in \mathbb{N}$ and $n \geq r$, we get

i) $a^{\xi(n-r)} b^{1-\xi(n-r)} \tilde{q}_{n-r} \tilde{q}_{n+r}-a^{\xi(n)} b^{1-\xi(n)} \tilde{q}_{n}^{2}=(-1)^{n+1-r} a^{\xi(r)} b^{1-\xi(r)}(1+h a b-a b) q_{r}^{2}$,

ii) $\left(\frac{b}{a}\right)^{\xi(n+r)} \tilde{l}_{n-r} \tilde{l}_{n+r}-\left(\frac{b}{a}\right)^{\xi(n)} \tilde{l}_{n}^{2}=(-1)^{n-r}\left(\frac{b}{a}\right)^{1-\xi(r)}(1+h a b-a b)(a b+4) q_{r}^{2}$.

Proof. i) From Theorem 1, we have

$$
\begin{gathered}
a^{\xi(n-r)} b^{1-\xi(n-r)} \tilde{q}_{n-r} \tilde{q}_{n+r}=\frac{a}{(a b)^{n-1}}\left(\frac{\tilde{\alpha}^{2} \alpha^{2 n}-\tilde{\alpha} \tilde{\beta} \alpha^{n-r} \beta^{n+r}-\tilde{\beta} \tilde{\alpha} \alpha^{n+r} \beta^{n-r}+\tilde{\beta}^{2} \beta^{2 n}}{(\alpha-\beta)^{2}}\right), \\
a^{\xi(n)} b^{1-\xi(n)} \tilde{q}_{n}^{2}=\frac{a}{(a b)^{n-1}}\left(\frac{\tilde{\alpha}^{2} \alpha^{2 n}-\tilde{\alpha} \tilde{\beta}(\alpha \beta)^{n}-\tilde{\beta} \tilde{\alpha}(\alpha \beta)^{n}+\tilde{\beta}^{2} \beta^{2 n}}{(\alpha-\beta)^{2}}\right) .
\end{gathered}
$$


From the properties of split complex numbers, we write $\tilde{\alpha} \tilde{\beta}=\tilde{\beta} \tilde{\alpha}=$ $1+h a b-a b$. Then, by using equation (4), we have

$$
\begin{aligned}
a^{\xi(n-r)} b^{1-\xi(n-r)} \tilde{q}_{n-r} \tilde{q}_{n+r}-a^{\xi(n)} b^{1-\xi(n)} \tilde{q}_{n}^{2} & =-\frac{a}{(a b)^{n-1}}(-a b)^{n-r}(1+h a b-a b)\left(\frac{\alpha^{r}-\beta^{r}}{\alpha-\beta}\right)^{2} \\
& =(-1)^{n+1-r} a^{\xi(r)} b^{1-\xi(r)}(1+h a b-a b) q_{r}^{2}
\end{aligned}
$$

ii) The proof can be done analogously to $i$.

Note that for $r=1$ in Theorem 5, we obtain the following result which are Cassini's identities for the SCFLN.

Corollary 1. For any positive integer $n$, we have

i) $a^{\xi(n-1)} b^{1-\xi(n-1)} \tilde{q}_{n-1} \tilde{q}_{n+1}-a^{\xi(n)} b^{1-\xi(n)} \tilde{q}_{n}^{2}=a(-1)^{n}(1+h a b-a b)$,

ii) $\left(\frac{b}{a}\right)^{\xi(n+1)} \tilde{l}_{n-1} \tilde{l}_{n+1}-\left(\frac{b}{a}\right)^{\xi(n)} \tilde{l}_{n}^{2}=(-1)^{n-1}(1+h a b-a b)(a b+4)$.

Note that for $r=n$ in Theorem 5, we obtain the following result.

Corollary 2. For any positive integer $n$, we have

i) $h a b \tilde{q}_{2 n}-a^{\xi(n)} b^{1-\xi(n)} \tilde{q}_{n}^{2}=-a^{\xi(n)} b^{1-\xi(n)}(1+h a b-a b) q_{n}^{2}$,

ii) $(h a b+2) \tilde{l}_{2 n}-\left(\frac{b}{a}\right)^{\xi(n)} \tilde{l}_{n}^{2}=\left(\frac{b}{a}\right)^{1-\xi(n)}(1+h a b-a b)(a b+4) q_{n}^{2}$.

Following Theorem gives D'ocagne identities for the SCFLN;

Theorem 6. For $m \geq n$ and $m, n \in \mathbb{N}$, we obtain

i) $a^{\xi(m n+m)} b^{\xi(m n+n)} \tilde{q}_{m} \tilde{q}_{n+1}-a^{\xi(m n+n)} b^{\xi(m n+m)} \tilde{q}_{m+1} \tilde{q}_{n}=(-1)^{n} a^{\xi(m-n)}(1+h a b-a b) q_{m-n}$,

ii) $a^{\xi(m n+n)} b^{\xi(m n+m)} \tilde{l}_{m} \tilde{l}_{n+1}-a^{\xi(m n+m)} b^{\xi(m n+n)} \tilde{l}_{m+1} \tilde{l}_{n}=(-1)^{n+1} a^{\xi(m-n)}(1+h a b-a b)(a b+4) q_{m-n}$.

Proof. $\quad$ i) From Theorem 1, we have

$$
\begin{aligned}
& a^{\xi(m n+m)} b^{\xi(m n+n)} \tilde{q}_{m} \tilde{q}_{n+1}=\frac{a(a b)^{-n}}{(a b)^{\frac{m-n-\xi(m-n)}{2}}}\left(\frac{\tilde{\alpha}^{2} \alpha^{m+n+1}-\tilde{\alpha} \tilde{\beta} \alpha^{m} \beta^{n+1}-\tilde{\beta} \tilde{\alpha} \alpha^{n+1} \beta^{m}+\tilde{\beta}^{2} \beta^{m+n+1}}{(\alpha-\beta)^{2}}\right), \\
& a^{\xi(m n+n)} b^{\xi(m n+m)} \tilde{q}_{m+1} \tilde{q}_{n}=\frac{a(a b)^{-n}}{(a b)^{\frac{m-n-\xi(m-n)}{2}}}\left(\frac{\tilde{\alpha}^{2} \alpha^{m+n+1}-\tilde{\alpha} \tilde{\beta} \alpha^{m+1} \beta^{n}-\tilde{\beta} \tilde{\alpha} \alpha^{n} \beta^{m+1}+\tilde{\beta}^{2} \beta^{m+n+1}}{(\alpha-\beta)^{2}}\right) .
\end{aligned}
$$

Let us label the left-hand side of the equality in $i$ as LHS. Then, from equation (4), we write

$$
\begin{aligned}
L H S & =\frac{a(a b)^{-n}}{(a b)^{\left\lfloor\frac{m-n}{2}\right\rfloor}} \tilde{\alpha} \tilde{\beta}(\alpha \beta)^{n}\left(\frac{\alpha^{m-n}-\beta^{m-n}}{\alpha-\beta}\right) \\
& =(-1)^{n} a^{\xi(m-n)}(1+h a b-a b) q_{m-n} .
\end{aligned}
$$

ii) The proof can be done analogously to $i$. 
We give summation formulas for the SCFLN in the following theorem.

Theorem 7. For $m, n \in \mathbb{Z}$, we have

i) $\left(\frac{b}{a}\right)^{\xi(m+1) \xi(n)} \tilde{q}_{m} \tilde{l}_{n}+\left(\frac{b}{a}\right)^{\xi(m) \xi(n+1)} \tilde{q}_{n} \tilde{l}_{m}=2 \tilde{q}_{m+n}+2 h a^{1-\xi(m+n)} b^{\xi(m+n)} \tilde{q}_{m+n+1}$,

ii) $(a b+4)\left(\frac{b}{a}\right)^{\xi(m+1) \xi(n+1)} \tilde{q}_{m} \tilde{q}_{n}+\left(\frac{b}{a}\right)^{\xi(m) \xi(n)} \tilde{l}_{m} \tilde{l}_{n}=2 \tilde{l}_{m+n}+2 h a^{\xi(m+n)} b^{1-\xi(m+n)} \tilde{l}_{m+n+1}$.

Proof. $\quad i)$ The proof can be done analogously to $i$.

ii) We must express that the proof should be examined for both cases of $m$ and $n$.

If both of $m$ and $n$ are even, from equations $(15),(16)$ and $(11)$, we find

$$
\begin{aligned}
(a b+4)\left(\frac{b}{a}\right) \tilde{q}_{m} \tilde{q}_{n}+\tilde{l}_{m} \tilde{l}_{n} & =(a b+4)\left(\frac{b}{a}\right)\left(q_{m} q_{n}+h a q_{m} q_{n+1}+h a q_{n} q_{m+1}+a^{2} q_{m+1} q_{n+1}\right) \\
& +l_{m} l_{n}+h b l_{m} l_{n+1}+h b l_{n} l_{m+1}+b^{2} l_{m+1} l_{n+1} \\
& =2 l_{m+n}+4 h b l_{m+n+1}+2 a b l_{m+n+2} \\
& =2 \tilde{l}_{m+n}+2 h b \tilde{l}_{m+n+1} .
\end{aligned}
$$

If both of $m$ and $n$ are odd, from equations 15,16 and 11 , we get

$$
\begin{aligned}
(a b+4) \tilde{q}_{m} \tilde{q}_{n}+\left(\frac{b}{a}\right) \tilde{l}_{m} \tilde{l}_{n} & =(a b+4)\left(q_{m} q_{n}+h b q_{m} q_{n+1}+h h q_{n} q_{m+1}+b^{2} q_{m+1} q_{n+1}\right) \\
& +\left(\frac{b}{a}\right)\left(l_{m} l_{n}+h a l_{m} l_{n+1}+h a l_{n} l_{m+1}+a^{2} l_{m+1} l_{n+1}\right) \\
& =2 l_{m+n}+4 h b l_{m+n+1}+2 a b l_{m+n+2} \\
& =2 \tilde{l}_{m+n}+2 h b \tilde{b}_{m+n+1} .
\end{aligned}
$$

If one of $m$ and $n$ is even and the other is odd, from equations (15), 16p and (11), we obtain

$$
\begin{aligned}
(a b+4) \tilde{q}_{m} \tilde{q}_{n}+\tilde{l}_{m} \tilde{l}_{n} & =2 l_{m+n}+4 h a l_{m+n+1}+2 a b l_{m+n+2} \\
& =2 \tilde{l}_{m+n}+2 h a \tilde{l}_{m+n+1} .
\end{aligned}
$$

If we put the all results together, we obtain the desired equation.

If we take $m=0$ in Theorem [7, it is easy to see the following:

Corollary 3. For $n \in \mathbb{Z}$, we have

i) $b \tilde{q}_{n}+\left(\frac{b}{a}\right)^{\xi(n)} \tilde{l}_{n}=2\left(\frac{b}{a}\right)^{\xi(n)} \tilde{q}_{n+1}$,

ii) $(a b+4)\left(\frac{b}{a}\right)^{\xi(n+1)} \tilde{q}_{n}+b \tilde{l}_{n}=2\left(\frac{b}{a}\right)^{\xi(n+1)} \tilde{l}_{n+1}$.

If we take $m=n$ in Theorem 7 it is easy to see the following: 
Corollary 4. For $n \in \mathbb{Z}$, we have

i) $\tilde{q}_{n} \tilde{l}_{n}=\tilde{q}_{2 n}+h a \tilde{q}_{2 n+1}$,

ii) $(a b+4)\left(\frac{b}{a}\right)^{1-\xi(n)} \tilde{q}_{n}^{2}+\left(\frac{b}{a}\right)^{\xi(n)} \tilde{l}_{n}^{2}=2 \tilde{l}_{2 n}+2 h b \tilde{l}_{2 n+1}$.

If we take $m=1$ in Theorem [7, it is easy to see the following:

Corollary 5. For $n \in \mathbb{Z}$, we have

i) $\tilde{q}_{1} \tilde{l}_{n}+\left(\frac{b}{a}\right)^{\xi(n+1)} \tilde{q}_{n} \tilde{l}_{1}=2 \tilde{q}_{n+1}+2 h a^{\xi(n)} b^{\xi(n+1)} \tilde{q}_{n+2}$,

ii) $(a b+4) \tilde{q}_{1} \tilde{q}_{n}+\left(\frac{b}{a}\right)^{\xi(n)} \tilde{l}_{1} \tilde{l}_{n}=2 \tilde{l}_{n+1}+2 h a^{\xi(n+1)} b^{\xi(n)} \tilde{l}_{n+2}$.

\section{Conclusion}

In this paper, we define split complex bi-periodic Fibonacci and Lucas numbers and give some properties of these new numbers. Thus, it is obtained a new genaralization for the split complex number sequences that have the similar recurrence relation. That is, in the all results of Section 2, we can express certain and immediate relationships as follows:

- If we replace $a=b=1$ in $\tilde{q}_{n}$ and $\tilde{l}_{n}$, we get the same result in 11 for the split complex Fibonacci and Lucas numbers.

- If we replace $a=b=2$ in $\tilde{q}_{n}$ and $\tilde{l}_{n}$, we find the split complex Pell and Pell-Lucas numberss.

- If we replace $a=b=k$ in $\tilde{q}_{n}$ and $\tilde{l}_{n}$, we obtain the split complex $k$-Fibonacci and $k$-Lucas numbers.

Declaration of Competing Interest The author declare that there are no conflicts of interest regarding the publication of this paper.

\section{REFERENCES}

[1] Aydin, F. T., Hyperbolic Fibonacci sequence, Universal Journal of Mathematics and Applications, 2(2) (2019), 59-64. https://doi.org/10.32323/ujma.473514

[2] Bala, A., Verma, V., Some properties of bi-variate bi-periodic Lucas polynomials, Annals of the Romanian Society for Cell Biology, 25(4) (2021), 8778-8784. https://www.annalsofrscb.ro/index.php/journal/article/view/3598.

[3] Bilgici, G., Two generalizations of Lucas sequence, Applied Mathematics and Computation, 245 (2014), 526-538. https://doi.org/10.1016/j.amc.2014.07.111.

[4] Catoni, F., Boccaletti, R., Cannata, R., Catoni, V., Nichelatti, E., Zampatti, P., The Mathematics of Minkowski Space-Time, Birkhauser, Basel, 2008.

[5] Dikmen, C.M., Hyperbolic Jacobsthal Numbers, Asian Research Journal of Mathematics, (2019) 1-9. https://doi.org/10.9734/arjom/2019/v15i430153

[6] Edson, M., Yayenie, O., A new generalization of Fibonacci sequences and extended Binet's Formula, Integers, 9(A48) (2009), 639-654. https://doi.org/10.1515/INTEG.2009.051

[7] Gargoubi, H., Kossentini, S., f-algebra structure on hyperbolic numbers, Adv. Appl. Clifford Algebr., 26(4) (2016), 1211-1233. https://doi.org/10.1007/s00006-016-0644-3

[8] Koshy, T., Fibonacci and Lucas Numbers with Applications, John Wiley and Sons Inc, NY, 2001. 
[9] Khadjiev, D., Goksal Y., Applications of hyperbolic numbers to the invariant theory in two-dimensional pseudo-Euclidean space, Adv. Appl. Clifford Algebr., 26 (2016), 645-668. https://doi.org/10.1007/s00006-015-0627-9

[10] Khrennikov, A., Segre, G., An Introduction to Hyperbolic Analysis, arxiv, 2005. http://arxiv.org/abs/math-ph/0507053v2.

[11] Motter, A. E, Rosa, A. F., Hyperbolic calculus, Adv. Appl. Clifford Algebr., 8(1) (1998), 109-128.

[12] Sobczyk, G., The hyperbolic number plane, The College Mathematics Journal, 26(4) (1995), 268-280.

[13] Soykan, Y., On hyperbolic numbers with generalized Fibonacci numbers components, preprint, (2019).

[14] Soykan, Y., Gocen, M., Properties of hyperbolic generalized Pell numbers, Notes on Number Theory and Discrete Mathematics, 26(4) (2020), 136-153. https://doi.org/10.7546/nntdm.2020.26.4.136-153

[15] Tan, E., Leung, H.H., Some basic properties of the generalized bi-periodic Fibonacci and Lucas sequences, Advances in Difference Equations, 2020.1 (2020), 1-11. https://doi.org/10.1186/s13662-020-2507-4

[16] Tasyurdu, Y., Hyperbolic Tribonacci and Tribonacci-Lucas Sequences, International Journal of Mathematical Analysis, 13(12) (2019), 565-572. https://doi.org/10.12988/ijma.2019.91167

[17] Verma, V., Bala, A., On properties of generalized bi-variate bi-periodic Fibonacci polynomials, International Journal of Advanced Science and Technology, 29(3) (2020), 8065-8072.

[18] Yayenie, O., A note on generalized Fibonacci sequence, Applied Mathematics and Computation, 217(12) (2011), 5603-5611. https://doi.org/10.1016/j.amc.2010.12.038 